\title{
ASSOCIAÇÃO DOS COAGULANTES TANFLOC SG E Moringa Oleífera COMO ALTERNATIVAS AO TRATAMENTO DE ÁGUA
}

\author{
B. A. M. OLIVEIRA ${ }^{1}$, J. M. MELO ${ }^{1}$, R. S. CASTRO ${ }^{1}$, H. H. D. GOES ${ }^{1}$ e J. D. P. \\ THEODORO $^{2}$ \\ ${ }^{1}$ Universidade Tecnológica Federal do Paraná \\ ${ }^{2}$ Universidade Tecnológica Federal do Paraná, Departamento de Engenharia Ambiental \\ E-mail para contato: bmeneghel.bm@gmail.com
}

\begin{abstract}
RESUMO - Devido à necessidade de garantir a qualidade das águas buscou-se através do uso simultâneo dos coagulantes orgânicos tanino (Tanfloc SG (50\%)) e extração salina da semente de Moringa oleífera (50\%) para avaliar os parâmetros turbidez, cor aparente e $\mathrm{pH}$, afim de constatar a eficiência do uso desses coagulantes nos processos de coagulação/floculação/sedimentação. Simulando as condições reais mais próximas de uma ETA, através do equipamento jar-teste, para as seguintes concentrações $5 \mathrm{mg} . \mathrm{L}^{-1}, 8 \mathrm{mg} . \mathrm{L}^{-1}$ e $10 \mathrm{mg} . \mathrm{L}^{-1}$ da mistura dos coagulantes naturais. Para o parâmetro turbidez nenhum dos ensaios dentre os dezoito apresentou taxa de remoção superior a 50\%, já para o parâmetro cor aparente para pelo menos um dos tempos de ensaio houve remoção superior a $50 \%$. Sendo assim, foi possível através desse procedimento foi possível constatar a necessidade de avaliar melhor o uso simultâneo desses coagulantes, para assim ser possível obter melhores resultados e avaliar a possibilidade de substituir os coagulantes inorgânicos.
\end{abstract}

\section{INTRODUÇÃO}

Devido às condições naturais e as ocasionadas pelas interferências dos seres humanos é de grande importância garantir a qualidade das águas. Sendo assim, existem as Estações de Tratamento de Água, onde para tratamento da água bruta ocorrem os processos de coagulação, floculação, sedimentação, filtração e desinfecção para posterior distribuição para população. Desta forma, devido ao uso atual de coagulantes químicos, pode ocorrer poluição devido à degradação desse material, sendo assim, faz-se necessário estudar uma forma alternativa de tratamento de água, utilizando os coagulantes orgânicos, já que esses são considerados biodegradáveis. Esse trabalho tem como objetivo analisar os parâmetros turbidez, cor aparente e pH para o uso simultâneo dos coagulantes tanino (Tanfloc SG (50\%)) e extração salina da semente de Moringa oleífera (50\%), almejando através deles alcançar eficiência nos processos de coagulação/floculação/sedimentação.

\section{METODOLOGIA}

A coleta da água de estudo foi realizada na SAMAE - Serviço Autônomo Municipal de Água e Esgoto, localizado no município de Ibiporã no estado do Paraná. A coleta foi realizada em um período de estiagem, desta forma, para as concentrações dos coagulantes foram 
utilizadas as indicadas por Trevisan (2014) para período de estiagem que são 5 mg.L $\mathrm{L}^{-1}, 8$ mg. $\mathrm{L}^{-1}$ e $10 \mathrm{mg} . \mathrm{L}^{1}$ para cada litro de amostra. Antes de iniciar os ensaios foi necessário fazer a correção do $\mathrm{pH}$, variando-se o pH obtém amostras ácidas $(5,30)$ e básicas $(9,06)$ para assim constatar se a variação de pH não afetará a ação dos coagulantes.

Para preparar a solução simultânea dos coagulantes naturais, inicialmente as mesmas foram preparadas separadamente. Para a solução de tanino (Tanfloc SG) pesou-se $1 \mathrm{~g}$ do coagulante, posteriormente transferido para um balão volumétrico e diluído em $1 \mathrm{~L}$ de água destilada, resultando em uma solução coagulante de concentração referente a $1 \mathrm{~g} . \mathrm{L}^{-1}$ de acordo com o procedimento realizado por Trevisan (2014). Em seguida, para preparar a solução salina de coagulante das sementes de Moringa Oleífera pesou-se $10 \mathrm{~g}$ da mesma já sem casca, essa quantidade foi triturada após a adição de $1 \mathrm{~L}$ de solução salina $(\mathrm{NaCl} 1 \mathrm{M})$. Preparou-se essa solução através da diluição de $58,44 \mathrm{~g}$ de cloreto de sódio $(\mathrm{NaCl})$ em $1 \mathrm{~L}$ de água destilada, obtendo-se a concentração de $1 \mathrm{M}$ para realizar a trituração das sementes. A fim de realizar a extração dos compostos ativos da Moringa Oleifera a solução foi filtrada, obtendose a solução coagulante na concentração de $10 \mathrm{~g} . \mathrm{L}^{-1}$, conforme sugerido por Bergamasco (2008).

Os ensaios foram baseados na aplicação do planejamento experimental Fatorial $3^{2}$ realizado em duplicata para os níveis $(-1,0,1)$. Para a realização dos 18 ensaios duas variáveis independentes foram estabelecidas, sendo elas $\mathrm{pH}$ e concentração de coagulante utilizada, tendo como variável resposta o $\mathrm{pH}$ final, cor aparente e turbidez. Para realizar as análises da forma mais próxima das condições reais de uma ETA os tempos e as velocidades de gradiente utilizados no do equipamento jar-teste são iguais aos padrões adotados pela SANEPAR. A sequência pode ser observada na Tabela 1:

Tabela 1. Tempos e velocidades de gradiente

\begin{tabular}{|c|c|c|c|c|c|c|c|c|c|}
\hline \multicolumn{2}{|c|}{$\begin{array}{l}\text { Mistura Rápida } \\
\text { Gradiente/Tempo }\end{array}$} & \multicolumn{2}{|c|}{$\begin{array}{c}\text { Mistura Lenta } 1 \\
\text { Gradiente/Tempo }\end{array}$} & \multicolumn{2}{|c|}{$\begin{array}{c}\text { Mistura Lenta } 2 \\
\text { Gradiente/Tempo }\end{array}$} & \multicolumn{2}{|c|}{$\begin{array}{c}\text { Mistura Lenta } 3 \\
\text { Gradiente/Tempo }\end{array}$} & \multicolumn{2}{|c|}{$\begin{array}{l}\text { Mistura Lenta } 4 \\
\text { Gradiente/Tempo }\end{array}$} \\
\hline $450 \mathrm{rpm}$ & $10 \mathrm{~s}$ & $90 \mathrm{rpm}$ & $120 \mathrm{~s}$ & $52 \mathrm{rpm}$ & $155 \mathrm{~s}$ & $40 \mathrm{rpm}$ & $160 \mathrm{~s}$ & $30 \mathrm{rpm}$ & $340 \mathrm{~s}$ \\
\hline
\end{tabular}

O jar-teste é composto por 6 jarros de 2 litros, sendo então necessário 12 litros de amostra para realizar as analises seguindo os parâmetros da Tabela 1. Ocorrendo coagulação e floculação para os tempos de mistura rápida e lenta respectivamente ocasionada pela presença de uma hélice em cada um dos jarros. As concentrações de coagulantes foram $5 \mathrm{mg} . \mathrm{L}^{-1}$ (2,5 de tanino + 2,5 de extrato de Moringa oleifera $), 8 \mathrm{mg} . \mathrm{L}^{-1}(4$ de tanino +4 de extrato de Moringa oleifera $)$ e $10 \mathrm{mg} . \mathrm{L}^{-1}(5$ de tanino +5 de extrato de Moringa oleifera $)$ para três variações de $\mathrm{pH}$ (neutro, básico e ácido), em duplicata. Após esse processo, foi necessário aguardar 30 minutos de sedimentação, onde a cada intervalo de 10 minutos ocorreu à coleta de amostra para avaliar em qual dos intervalos houve a melhor eficiência de remoção dos parâmetros cor aparente e turbidez.

\subsection{Cálculo das eficiências de remoção dos parâmetros}

Para o cálculo da taxa de remoção dos parâmetros cor aparente e turbidez em cada ensaio é usada a Equação 1. 
$E_{(x)}=\frac{P_{A B}-P_{E}}{P_{A B}} \times 100$

Onde:

$\mathrm{E}_{(\mathrm{x})}$ : Eficiência de remoção do parâmetro, no ensaio $\mathrm{x} ; \mathrm{P}_{\mathrm{AB}}$ : Valor do parâmetro medido na amostra bruta; $\mathrm{P}_{\mathrm{E}}$ : Valor do parâmetro medido no ensaio $\mathrm{X}$.

\section{RESULTADOS E DISCUSSÕES}

\subsection{Caracterização da Amostra}

A caracterização da amostra bruta em relação aos parâmetros cor aparente é $141 \mathrm{uH}$, turbidez é 45,8 uT e pH é 6,86. É possível constatar através da Tabela 1 os resultados para os parâmetros $\mathrm{pH}$, turbidez e cor aparente para os 18 ensaios.

Tabela 1. Valores dos parâmetros cor aparente, turbidez e pH obtidos nos ensaios com a solução coagulante 50\% Tanfloc SG e 50\% Moringa Oleífera.

\begin{tabular}{|c|c|c|c|c|c|c|c|c|c|c|c|}
\hline \multirow[b]{2}{*}{ Ensaio } & \multirow[b]{2}{*}{$\mathbf{p H}$} & \multirow[b]{2}{*}{$\begin{array}{l}\text { [Tanfloc } \\
+ \\
\text { Moringa] } \\
\text { mgL }^{-1}\end{array}$} & \multicolumn{3}{|c|}{ pH } & \multicolumn{3}{|c|}{ Turbidez (uT) } & \multicolumn{3}{|c|}{ Cor (uH) } \\
\hline & & & $10 \mathrm{~min}$ & $20 \mathrm{~min}$ & $30 \mathrm{~min}$ & $10 \mathrm{~min}$ & $20 \mathrm{~min}$ & $30 \mathrm{~min}$ & $10 \mathrm{~min}$ & $20 \mathrm{~min}$ & $30 \mathrm{~min}$ \\
\hline 1 & $-1(5,30)$ & $-1(5)$ & 5,01 & 5,14 & 5,66 & 20,7 & 16,9 & 15,8 & 43 & 39 & 40 \\
\hline 2 & $-1(5,30)$ & $0(8)$ & 5,07 & 5,1 & 5,64 & 20,4 & 17,8 & 16,3 & 42 & 47 & 44 \\
\hline 3 & $-1(5,30)$ & $1(10)$ & 5,11 & 5,11 & 5,44 & 23,7 & 18,7 & & 52 & 40 & 48 \\
\hline 4 & $0(6,86)$ & $-1(5)$ & 6,66 & 6,89 & 6,97 & 26 & 23,8 & 25,7 & 75 & 62 & 73 \\
\hline 5 & $0(6,86)$ & $0(8)$ & 6,64 & 6,89 & 6,97 & 28,4 & 23,8 & 25,7 & 75 & 62 & 73 \\
\hline 6 & $0(6,86)$ & $1(10)$ & 6,74 & 6,95 & 7,08 & 26 & 23,8 & 25,7 & 75 & 62 & 73 \\
\hline 7 & $1(9,06)$ & $-1(5)$ & 8,74 & 8,76 & 9,11 & 15 & 13,3 & 13,8 & 39 & 35 & 35 \\
\hline 8 & $1(9,06)$ & $0(8)$ & 9,13 & 8,76 & 9,07 & 26,1 & 22,1 & 21,3 & 73 & 67 & 67 \\
\hline 9 & $1(9,06)$ & $1(10)$ & 9,25 & 8,83 & 9,14 & 20,7 & 19,5 & 20,3 & 60 & 62 & 63 \\
\hline 10 & $-1(5,30)$ & $-1(5)$ & 5,16 & 5,17 & 5,42 & 21,2 & 15,4 & 15,2 & 44 & 41 & 40 \\
\hline 11 & $-1(5,30)$ & $0(8)$ & 5,05 & 5,16 & 5,37 & 25 & 19,6 & 16,4 & 51 & 49 & 45 \\
\hline 12 & $-1(5,30)$ & $1(10)$ & 5 & 5,14 & 5,32 & 20,5 & 20,6 & 15,3 & 49 & 50 & 47 \\
\hline 13 & $0(6,86)$ & $-1(5)$ & 6,74 & 6,95 & 7,08 & 25,2 & 24,4 & 24,2 & 76 & 69 & 72 \\
\hline 14 & $0(6,86)$ & $0(8)$ & 6,76 & 6,89 & 6,97 & 29 & 25,8 & 24,7 & 79 & 76 & 76 \\
\hline 15 & $0(6,86)$ & $1(10)$ & 6,79 & 6,84 & 7,05 & 28,5 & 23,4 & 23,2 & 70 & 76 & 73 \\
\hline 16 & $1(9,06)$ & $-1(5)$ & 9,44 & 8,99 & 9,11 & 14,4 & 14 & 12,7 & 37 & 34 & 35 \\
\hline 17 & $1(9,06)$ & $0(8)$ & 9,28 & 9,02 & 9,11 & 22,8 & 21,6 & 21,2 & 64 & 69 & 63 \\
\hline 18 & $1(9,06)$ & $1(10)$ & 9,51 & 9,08 & 9,11 & 24 & 22,7 & 22,5 & 69 & 74 & 69 \\
\hline
\end{tabular}


Aparentemente foi possível perceber que ocorreu diferença na cor aparente da água de estudo (Figura 2), além disso, também observou-se a formação dos flocos e sua sedimentação por meio da ação da gravidade.

Figura 2 - A: água bruta; B: água após o tratamento.
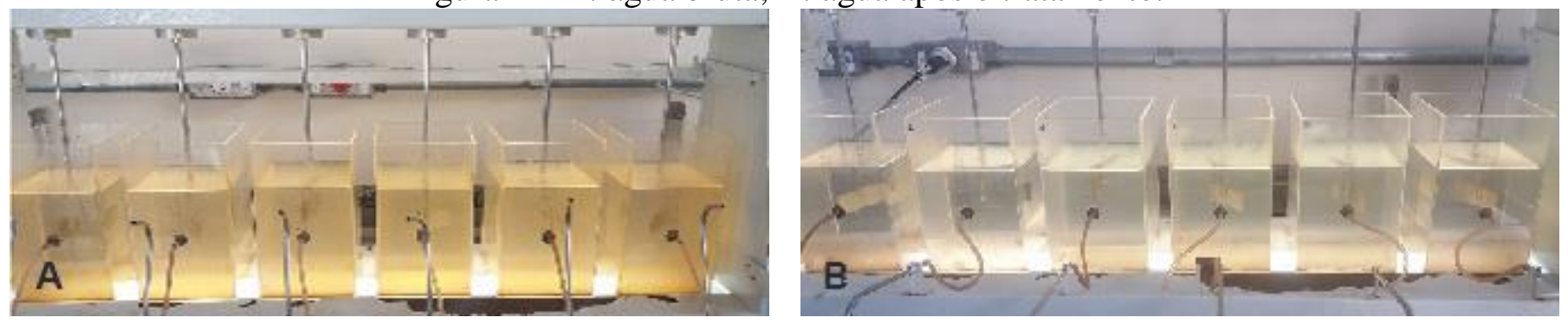

\subsection{Remoções dos Parâmetro Turbidez e Cor Aparente}

As Figuras 3 e 4 representam as taxa de remoções dos parâmetros turbidez e cor aparente dos coagulantes orgânicos usados simultaneamente Tanfloc SG (50\%) + Moringa Oleífera (50\%), respectivamente.

Figura 3. Taxa de remoção do parâmetro turbidez dos coagulantes orgânicos usados simultaneamente Tanfloc SG (50\%) + Moringa Oleífera (50\%).

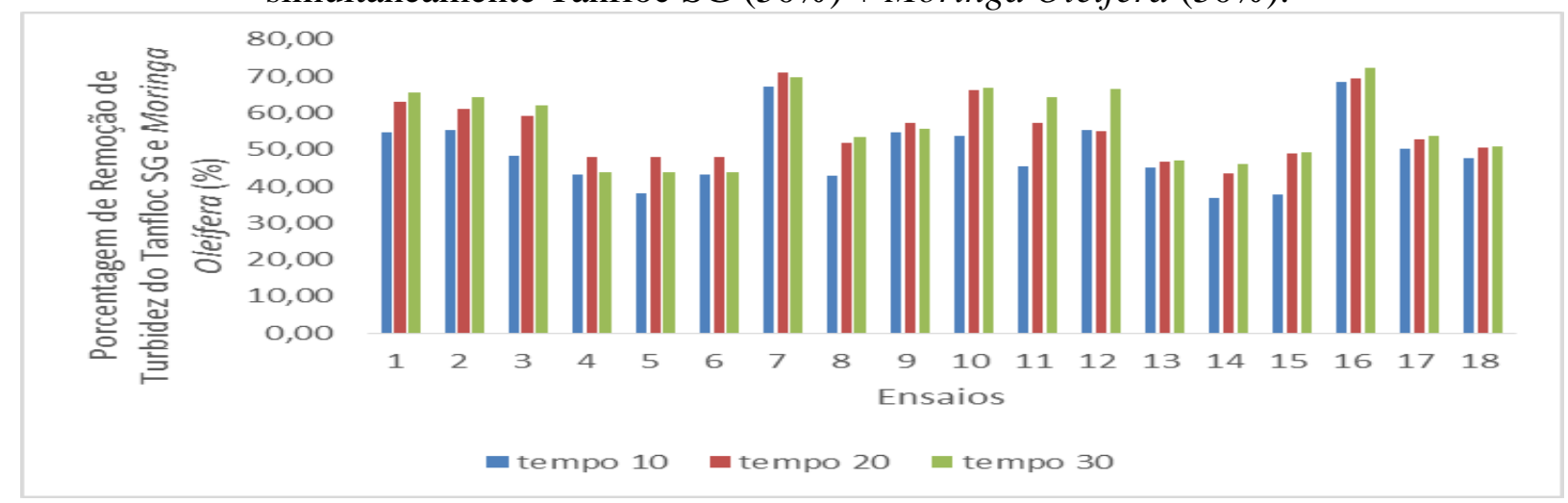

Figura 4. Taxa de remoção do parâmetro cor dos coagulantes orgânicos usados simultaneamente Tanfloc SG (50\%) + Moringa Oleífera (50\%).

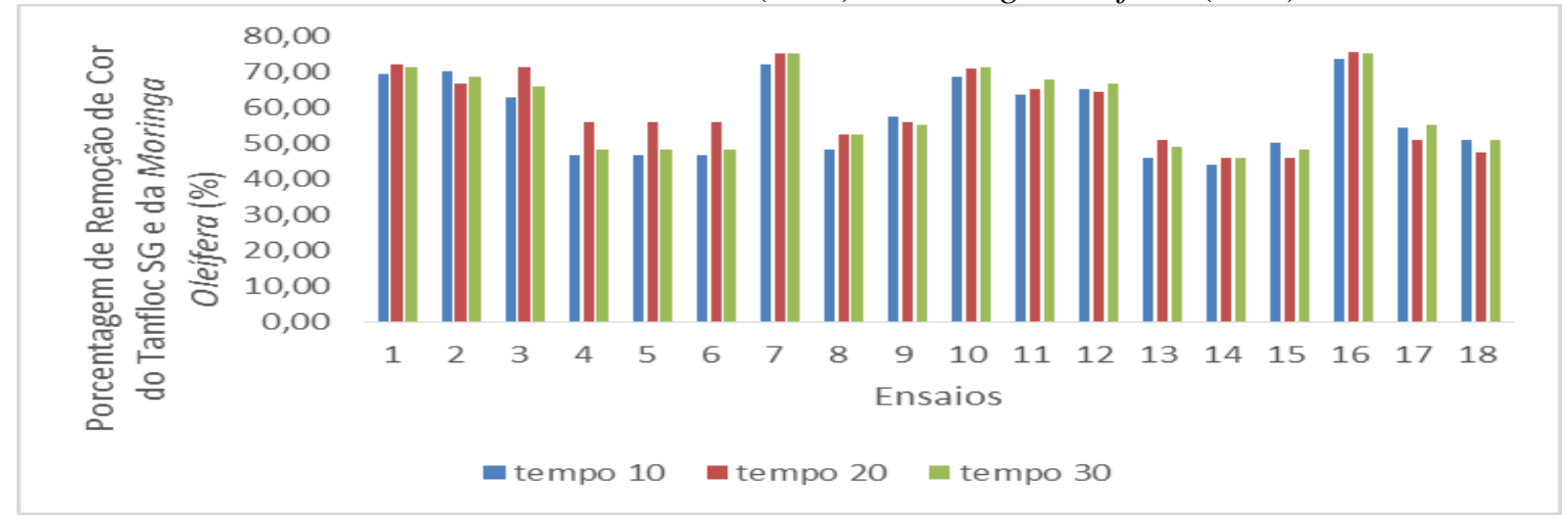


Para os coagulantes utilizados de forma simultânea, nenhum dos ensaios dentre os dezoito apresentou taxa de remoção superior a 50\% para o parâmetro turbidez, sendo o melhor resultado para o ensaio 15, que atingiu em 10 minutos uma eficiência de remoção de 28,5\%. Constatou-se que o ensaio 16 apresentou remoção de $12,7 \%$, sendo o pior resultado para o parâmetro turbidez (Figura 3). Para o parâmetro cor aparente percebe-se que a eficiência de remoção para os coagulantes associados apresentaram para doze ensaios $(3,4,5$, $6,8,9,11,13,14,15,17$, e 18) em pelo menos um dos tempos, eficiência de remoção maior que $50 \%$, porém nenhum teve taxa igual ou superior a $80 \%$ (Figura 4).

Trevisan (2014) constatou, para o coagulante tanino (Tanfloc SG) para as concentrações $5 \mathrm{mg} . \mathrm{L}^{-1}, 8 \mathrm{mg} . \mathrm{L}^{-1}$ e $10 \mathrm{mg} \cdot \mathrm{L}^{-1}$ as seguintes taxas de remoção para os parâmetros cor aparente e turbidez, respectivamente $84,6 \%$ e $88 \%$. Em relação à Moringa Oleífera, de acordo com Bergamasco (2008), também para uma época de estiagem foram utilizadas as concentrações 3 $\mathrm{mg} . \mathrm{L}^{-1}, 5 \mathrm{mg} . \mathrm{L}^{-1}$ e $7 \mathrm{mg} . \mathrm{L}^{-1}$, para esses valores esse coagulante não apresentou valores satisfatórios, o autor aponta como possível causa desses resultados a alta carga de matéria orgânica presente nesse coagulante e a baixa presença de sólidos em suspensão e dissolvidos afetando negativamente a remoção dos parâmetros cor aparente e turbidez. Para o presente trabalho nenhum dos ensaios atingiu taxa de remoção dos parâmetros turbidez e cor aparente superior ao valor de $80 \%$ como ocorreu com a utilização separada do coagulante tanino. Sendo assim, devido aos valores não satisfatórios do uso apenas da Moringa Oleífera acredita-se que uma das possíveis causas, seja como mencionado por Bergamasco (2008), a grande carga orgânica desse coagulante e a baixa presença de sólidos, visto que a época da coleta era de estiagem.

\subsection{Variação do pH da Água sob o Efeito dos Coagulantes Orgânicos}

Para os ensaios realizados não ocorreram variações significativas nos valores de $\mathrm{pH}$, pois para todos em os ensaios a faixa de $\mathrm{pH}$ permaneceu próxima ao mensurado antes do início das análises conforme mostra a Figura 5.

Figura 5. Variação do pH da amostra para os coagulantes orgânicos usados simultaneamente Tanfloc SG (50\%) + Moringa Oleífera (50\%).

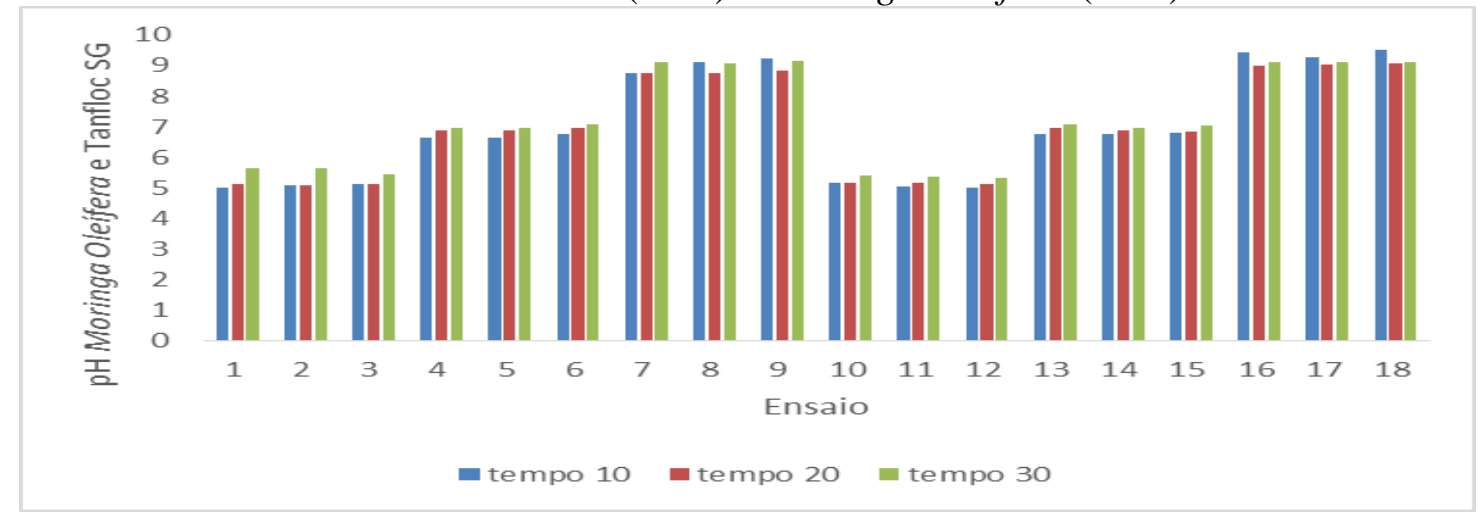

Em relação ao parâmetro pH, Trevisan (2014), afirma que as variações não são relevantes, pois não há consumo da alcalinidade do meio, não dependendo então da 
concentração utilizada. Nesse trabalho também se observou uma variação pouco significativa do $\mathrm{pH}$.

\section{CONCLUSÃO}

Com a realização desse trabalho foi possível constatar que o uso simultâneo dos coagulantes orgânicos tanino e Moringa Oleífera não apresentaram altas taxas de remoção para os parâmetros cor aparente e turbidez, sendo os melhores resultados para o parâmetro cor, visto que 12 ensaios $(3,4,5,6,8,9,11,13,14,15,17$, e 18) apresentaram resultados acima de $50 \%$ em contra partida nenhum ensaio para o parâmetro turbidez atingiu esse valor. Por fim, ainda se faz necessários estudos mais aprofundados para a realização dos processos de coagulação/floculação/sedimentação por meio da utilização de coagulantes orgânicos a fim de substituir os inorgânicos.

\section{REFERÊNCIAS}

BERGAMASCO, Rosângela et al. Otimização dos tempos de mistura e decantação no processo de coagulação/floculação da água bruta por meio da Moringa Oleífera Lam. Acta Scientiarum. Technology. Maringá, v. 30, 2008.

FAGAR, Importância da água. 2016. Disponível em:

<http://www.fagar.pt/index.php?option=com_content\&view=article\&id=52\&Itemid=76>. Acesso em: 11 mar 2017.

MACEDO, Jorge A. B. Águas \& Águas. $3^{\circ}$ ed. Belo Horizonte: Editora UFJF, 2007 SERVIÇO AUTONÔMO DE ÁGUA E ESGOTO (SAMAE). Disponível em: <http://www.samaeibi.com.br/conteudo_texto_8.html>. Acesso: 11 de mar 2017.

TREVISAN, Thales S. Coagulante Tanfloc SG como uma alternativa ao uso de coagulantes químicos no tratamento de água na ETA Cafezal. 2014. 106 f. Trabalho de Conclusão de Curso (Graduação) - Curso Superior de Engenharia Ambiental. Universidade Tecnológica Federal do Paraná, Londrina, 2014. 